LIPIDS

\title{
Remnant cholesterol is associated with ischaemic heart disease
}

An elevated plasma triglyceride level is associated with an increased risk of cardiovascular disease, but this association could be the result of confounding factors. Investigators from Denmark have, therefore, examined the nonfasting level of remnant cholesterol-the cholesterol content of triglyceride-rich lipoproteinsin a population-based cohort of 73,513 individuals. A genetically-determined increase in nonfasting remnant cholesterol level of $1 \mathrm{mmol} / \mathrm{l}$ was, indeed, associated with an increased risk of ischaemic heart disease (OR 2.8, 95\% CI 1.9-4.2).

An increased remnant cholesterol level is also associated with a decreased HDL-cholesterol level. To distinguish between these two risk factors and to remove potential confounding effects, study participants were genotyped for 15 alleles affecting either remnant cholesterol, remnant cholesterol and HDL cholesterol,
HDL cholesterol alone, or LDL cholesterol. Alleles associated with increased remnant cholesterol (alone or in combination with decreases in HDL cholesterol) or LDL cholesterol were associated with ischaemic heart disease, whereas alleles associated with a decreased HDL-cholesterol level were not.

The number of risk alleles for remnant cholesterol or LDL cholesterol was more strongly associated with the risk of ischaemic heart disease than were biochemical lipid measurements. Given that genetic determinants of lipid levels act over the lifetime of an individual, they affect exposure to high lipid levels over many years.

Megan Cully

Original article Varbo, A. et al. Remnant cholesterol as a causal risk factor for ischemic heart disease. J. Am. Coll. Cardiol. doi:10.1016/j.jacc.2012.08.1026 